\title{
THE BOUNDEDNESS OF ORTHONORMAL POLYNOMIALS ON CERTAIN CURVES OF THE FOURTH DEGREE
}

\author{
BY \\ DUNHAM JACKSON(1)
}

1. Introduction. For the study of the boundedness of systems of orthonormal polynomials on algebraic curves, a property intimately associated with the convergence of the corresponding developments in series, various elementary devices and expedients have been found useful under one set of circumstances or another. Among these are: (a) exploitation of geometric symmetry of the domain of integration, (b) linear transformation of the figure, (c) parametric representation, (d) permutation of the functions to which the Schmidt process is applied, (e) replacement of these functions by suitable linear combinations of them (ultimately a generalization of the preceding), (f) application of the theory of orthogonal polynomials satisfying auxiliary conditions [4] $\left({ }^{2}\right)$. Use of Korous's theorem [12, p. 157], [5, p. 205208], [6], [7] is pertinent in every situation, to replace a particular weight function by a more general one, or to substitute a simpler weight function for a more complicated one resulting from the exigencies of the initial approach to an assigned problem. Of the special methods listed, (a) has been used in connection with certain rectilinear figures [10], [11], (a) and (b) have produced a considerable amount of information with regard to orthonormal systems on the ellipse, hyperbola, and parabola [7], (a), (c), (d), and (f) have been applied to loci of the form $y=x^{m}$, in which $m$ is an integer or a sufficiently simple fraction [8], and (a), (c), (e), and (f) have been brought to bear on selected curves of the third degree not previously dealt with [9].

The general problem is of such a degree of complexity that there is no reason to expect that it can be dealt with by any combination of the special devices referred to. On the other hand, even if attention is limited to these particular expedients, any attempt at an exhaustive account of their possibilities in permutation and combination would be totally unmanageable. It seems still worth while to proceed at least a little further with the analysis of illustrative examples.

In this spirit the present paper discusses certain rational curves of the fourth degree in parametric representation, with somewhat more elaborate development of (e) than has been called for in previous instances, and with special emphasis on the curve $x^{4}+y^{4}=1$ (previously studied from a different point of view, see [3]), the treatment being based on (a), (b), and (c), with

Presented to the Society, December 29, 1946; received by the editors January 21, 1947.

(1) Deceased November 6, 1946.

(2) Numbers in brackets refer to the bibliography at the end of the paper. 
a parametric representation in terms of trigonometric functions and further auxiliary change of variables.

2. The curve $y=A x^{4}+B x^{3}+C x^{2}+D x+E$. As in connection with the corresponding curve of the third degree, the formulas can be somewhat simplified by means of a preliminary linear transformation of coordinates. This procedure does not however immediately reduce the problem to a form previously considered, as it did in the case of the curve of lower degree [8], and the significance of the comparatively novel element in the discussion, the one listed under (e) above, appears to be brought out more clearly if the equation is left in its general form. It is understood that $A \neq 0$, otherwise one of the discussions of curves of lower degree wou'd be applicable. The variable $x$ is to range over an arbitrary interval $a \leqq x \leqq b$.

Since any monomial containing $x^{4}$ as a factor reduces on the curve to an expression of lower degree, the system of orthogonal polynomials for the curve is constructed from the monomials

$$
1 ; x, y ; x^{2}, x y, y^{2} ; x^{3}, x^{2} y, x y^{2}, y^{3} ; x^{3} y, x^{2} y^{2}, x y^{3}, y^{4} ; \cdots .
$$

In terms of $x$ as parameter the orthonormal polynomials are obtained by application of the Schmidt process to the sequence of functions

$$
\begin{aligned}
& 1 ; x, A x^{4}+B x^{3}+C x^{2}+D x+E ; x^{2}, x\left(A x^{4}+B x^{3}+C x^{2}+D x+E\right), \\
& \left(A x^{4}+B x^{3}+C x^{2}+D x+E\right)^{2} ; x^{3}, x^{2}\left(A x^{4}+B x^{3}+C x^{2}+D x+E\right), \\
& x\left(A x^{4}+B x^{3}+C x^{2}+D x+E\right)^{2},\left(A x^{4}+B x^{3}+C x^{2}+D x+E\right)^{3} ; \cdots
\end{aligned}
$$

Let $q_{0}(x), q_{1}(x), q_{2}(x), \cdots$ be the orthonormal polynomials in $x$ formed from this sequence in the order indicated. Their degrees in terms of $x$ are respectively at most

$$
0 ; 1,4 ; 4,5,8 ; 8,8,9,12 ; 12,12,13,16 ; \cdots \text {; }
$$

or, with a margin of actual inequality in most cases, do not exceed

$$
3 ; 4,5 ; 6,7,8 ; 9,10,11,12 ; 13,14,15,16 ; \cdots \text {. }
$$

If $p_{0}(x), p_{1}(x), p_{2}(x), \cdots$ are the normalized Legendre polynomials for the interval $(a, b)$, each $q_{n}(x)$ is expressible as a linear combination of $p_{0}(x)$, $p_{1}(x), \cdots, p_{n+3}(x)$.

If $P(x)$ is any polynomial which is linearly expressible in terms of the first $r+1$ members of the sequence (2),

$$
y P(x)=\left(A x^{4}+B x^{3}+C x^{2}+D x+E\right) P(x)
$$

is linearly expressible in terms of the first $r+5$ members of the same sequence, since the product of any individual member of the sequence by $y$ is one of the next four members. If $P(x)$ is linearly expressible in terms of the first $r+1$ members of the sequence, and if $x P(x), x^{2} P(x), x^{8} P(x)$ are each linearly ex- 
pressible in terms of the first $r+5$ members, it follows that $x^{4} P(x)$ is similarly expressible in terms of the first $r+5$ members. In other words, if $P(x)$ is a linear combination of $q_{0}, q_{1}, \cdots, q_{r}$, being then a linear combination of the first $r+1$ members, and if each of the products $x P(x), x^{2} P(x), x^{3} P(x)$ is a linear combination of $q_{0}, q_{1}, \cdots, q_{r+4}$, then $x^{4} P(x)$ is a linear combination of $q_{0}, q_{1}, \cdots, q_{r+4}$.

Suppose in particular that $x^{4 j}$ is a linear combination of $q_{0}, q_{1}, \cdots, q_{4 j+2}$, and that $x^{4 j+1}, x^{4 j+2}, x^{4 j+3}$ are linear combinations of $q_{0}, q_{1}, \cdots, q_{4 j+4}$; $q_{0}, q_{1}, \cdots, q_{4 j+5} ; q_{0}, q_{1}, \cdots, q_{4 j+6}$ respectively. Then the hypotheses of the statement in italics are fulfilled for $P(x)=x^{4 j}$ with $r=4 j+2$. It can be inferred that $x^{4 j+4}$ is a linear combination of $q_{0}, q_{1}, \cdots, q_{4 j+6}$. Furthermore, the hypotheses are now fulfilled for $P(x)=x^{4 j+1}$ with $r=4 j+4$. Hence $x^{4 j+5}$ is linearly expressible in terms of $q_{0}, q_{1}, \cdots, q_{4 j+8}$. The argument can be applied again to the function $P(x)=x^{4 j+2}$, with $r=4 j+5$, to show that $x^{4 j+6}$ is linearly expressible in terms of $q_{0}, q_{1}, \cdots, q_{4 j+9}$, and then to $P(x)=x^{4 j+3}$, with $r=4 j+6$, with the conclusion that $x^{4 i+7}$ is linearly expressible in terms of $q_{0}, q_{1}, \cdots, q_{4 j+10}$. In summary, the conditions enumerated in the first sentence of the present paragraph are met if $j$ is replaced by $j+1$, and the conclusions can be advanced accordingly.

It is apparent by inspection that the conditions are satisfied for $j=0$, with a margin of inequality in the comparison of indices except in the case of $x^{3}$, since $1, x, x^{2}, x^{3}$ are themselves the first, second, fourth and seventh members of the sequence (2), and so expressible in terms of $q_{0} ; q_{0}, q_{1} ; q_{0}, \cdots, q_{3}$; and $q_{0}, \cdots, q_{6}$ respectively. They are satisfied consequently for $j=1$, and thence by induction for arbitrary positive integral $j$. In a single brief statement, $x^{k}$ is linearly expressible in terms of $q_{0}, q_{1}, \cdots, q_{k+3}$ for $k=0,1,2$, $3, \cdots$.

By the fundamental property of the $q$ 's, therefore, $q_{n}(x)$ is orthogonal to $x^{k}$ over the interval $(a, b)$, and orthogonal to every polynomial of the $k$ th degree, if $n>k+3$. When $q_{n}(x)$ is expressed as a linear combination of $p_{0}(x), \cdots, p_{n+3}(x)$, the coefficient of $p_{k}$ vanishes for $k<n-3$. The representation has the form

$$
q_{n}(x)=\sum_{k=n-3}^{n+3} c_{n k} p_{k}(x)
$$

with

$$
c_{n k} \doteq \int_{a}^{b} q_{n}(x) p_{k}(x) d x .
$$

Since the number of terms has the fixed upper bound 7 as $n$ increases, since

$$
c_{n k}^{2} \leqq \int_{a}^{b}\left[q_{n}(x)\right]^{2} d x \int_{a}^{b}\left[p_{k}(x)\right]^{2} d x=1,
$$


and since the p's are uniformly bounded throughout any closed interval interior to $(a, b)$, the $q$ 's are similarly bounded.

This to be sure is for the orthonormal system defined with unit weight function for integration with respect to $x$. Since however the factor $d s / d x$ satisfies the conditions appropriate for the application of Korous's theorem, if $s$ is arc length, the conclusion is equally valid with unit weight function or any equivalent weight function and integration with respect to $s[7, \mathrm{pp}$. 352-353].

3. The curve $x=t^{2}, y=t^{4}+t$. This is a rational curve having no singular point at finite distance. Its shape is most easily recognized from the equation $y=x^{2} \pm x^{1 / 2}$; it is real for $x \geqq 0$. In terms of integral powers of $x$ and $y$ the equation is

$$
x^{4}-2 x^{2} y+y^{2}-x=0 .
$$

Any monomial containing $x^{4}$ as a factor can be expressed on the curve by a combination of terms of lower degree, and the sequence (1) can be taken as basis for the construction of the orthonormal system.

In terms of $t$ the sequence becomes

$$
1 ; t^{2}, t^{4}+t ; t^{4}, t^{2}\left(t^{4}+t\right),\left(t^{4}+t\right)^{2} ; t^{6}, t^{4}\left(t^{4}+t\right), t^{2}\left(t^{4}+t\right)^{2},\left(t^{4}+t\right)^{3} ; \cdots
$$

Let $q_{0}(t), q_{1}(t), q_{2}(t), \cdots$ be the orthonormal polynomials formed from the functions of this sequence in order, for an arbitrary interval $a \leqq t \leqq b$. Their respective degrees are at most

$$
0 ; 2,4 ; 4,6,8 ; 8,8,10,12 ; 12,12,14,16 ; \cdots,
$$

each entry in (1) from $x^{2} y$ on being the product of the fourth preceding by $y=t^{4}+t$. The degree of $q_{n}(t)$ does not exceed $n+3$, and if $p_{0}(t), p_{1}(t), p_{2}(t), \cdots$ are the normalized Legendre polynomials in $t$ for the interval $a \leqq t \leqq b, q_{n}(t)$ is a linear combination of $p_{0}(t), p_{1}(t), \cdots, p_{n+3}(t)$.

By continuation of the argument of the last section, as adapted to the present situation, if $P(t)$ is a linear combination of $q_{0}(t), q_{1}(t), \cdots, q_{r}(t)$, $y P(t)=\left(t^{4}+t\right) P(t)$ is a linear combination of $q_{0}, q_{1}, \cdots, q_{r+4}$, and if at the same time $t P(t)$ is a linear combination of $q_{0}, q_{1}, \cdots, q_{r+4}$, the same is true of $t^{4} P(t)$. The powers $1, t, t^{2}, t^{3}$, are linearly expressible respectively in terms of $q_{0} ; q_{0}, q_{1}, q_{2}, q_{3} ; q_{0}, q_{1} ; q_{0}, \cdots, q_{6}$ (a term in $q_{3}$ being required to eliminate $t^{4}$ from the expression for $t^{4}+t$ in terms of $q_{0}, q_{1}, q_{2}$, and a term in $q_{6}$ to eliminate $t^{6}$ from the expression for $\left.t^{2}\left(t^{4}+t\right)\right)$. If $t^{4 i}, t^{4 j+1}, t^{4 j+2}, t^{4 j+3}$ are linear combinations of $q_{0}, \cdots, q_{4 j+2} ; q_{0}, \cdots, q_{4 j+4} ; q_{0}, \cdots, q_{4 j+5} ; q_{0}, \cdots, q_{4 j+6}$ respectively, $t^{4 j+4}, t^{4 j+5}, t^{4 j+6}, t^{4 j+7}$ are successively seen to be linear combinations of $q_{0}, \cdots, q_{4 j+6} ; q_{0}, \cdots, q_{4 j+8} ; q_{0}, \cdots, q_{4 j+9} ;$ and $q_{0}, \cdots, q_{4 j+10}$. The induction is complete from $j$ to $j+1$, with many vanishing coefficients, to be sure, in the representations described. For each non-negative integral value of $k, t^{k}$ is a linear combination of $q_{0}(t), q_{1}(t), \cdots, q_{k+3}(t)$. The general polynomial 
$q_{n}(t)$ is orthogonal to each power $t^{k}$ with exponent less than $n-3$, and to ever polynomial of degree lower than $n-3$; it has a representation as a sum of not more than seven terms in the form

$$
q_{n}(t)=\sum_{k=n-3}^{n+3} c_{n k} p_{k}(t)
$$

with $\left|c_{n k}\right| \leqq 1$, and is uniformly bounded throughout any closed interval of values of $t$ interior to $(a, b)$.

4. The curve $x=t^{2}, y=t^{4}+t^{3}$. Real points are obtained once more for $x \geqq 0$. Alternative representations are $y=x^{2} \pm x^{3 / 2}$,

$$
x^{4}-x^{3}-2 x^{2} y+y^{2}=0 .
$$

There is a cusp at the origin with a horizontal tangent.

The fundamental monomials for the orthonormal system are still those of the sequence (1), or in terms of the parameter

(4) $1 ; t^{2}, t^{4}+t^{3} ; t^{4}, t^{2}\left(t^{4}+t^{3}\right),\left(t^{4}+t^{3}\right)^{2} ; t^{6}, t^{4}\left(t^{4}+t^{3}\right), t^{2}\left(t^{4}+t^{3}\right)^{2},\left(t^{4}+t^{3}\right)^{3}, \cdots$.

Clearly no linear combination of a finite number of these expressions will reduce to the first power of $t$. For this reason the orthonormal polynomials of the present problem will be expressed, not in terms of Legendre polynomials, but in terms of the orthonormal system formed for the interval $a \leqq t \leqq b$ under consideration from the successive powers of $t$ with omission of the first [8]. Let the polynomials of the latter system be denoted by $q_{0}(t), q_{2}(t), q_{3}(t), \cdots$, each being of the degree indicated by its subscript. The symbol $q_{1}(t)$ will be admitted on occasion for the sake of uniformity with the understanding that $q_{1}(t) \equiv 0$. Let the orthonormal polynomials corresponding to (4) be denoted by $r_{0}(t), r_{1}(t), r_{2}(t), \cdots$, with subscripts and degrees which are in general different. It will be seen presently that all the powers of $t$ are represented except the first.

The degrees of $r_{0}, r_{1}, \cdots$ respectively do not exceed

$$
0 ; 2,4 ; 4,6,8 ; 8,8,10,12 ; 12,12,14,16 ; \cdots \text {, }
$$

and in general the degree of $r_{n}(t)$ is at most $n+3$. Since $r_{n}(t)$ satisfies the auxiliary condition which characterizes the $q$ 's, namely that its first derivative with respect to $t$ vanishes for $t=0$, it can be linearly expressed in terms of the $q$ 's, and specifically in terms of $q_{0},\left[q_{1}\right], q_{2}, \cdots, q_{n+3}$.

The powers $t^{2}, t^{3}, t^{4}, t^{5}$ are linearly expressible in terms of $r_{0}, r_{1} ; r_{0}, r_{1}, r_{2}, r_{3}$; $r_{0}, r_{1}, r_{2}, r_{3} ; r_{0}, r_{1}, \cdots, r_{6}$. If $P(t)$ can be linearly expressed in terms of $r_{0}, \cdots, r_{m}$, and $t^{3} P(t)$ in terms of $r_{0}, \cdots, r_{m+4},\left(t^{4}+t^{3}\right) P(t)$ and hence $t^{4} P(t)$ can be linearly expressed in terms of $r_{0}, \cdots, r_{m+4}$. If $t^{4 j+2}, t^{4 j+8}, t^{4 j+4}$, $t^{4 j+5}$ can be linearly expressed in terms of $r_{0}, \cdots, r_{4 j+2} ; r_{0}, \cdots, r_{4 j+3}$; $r_{0}, \cdots, r_{4 j+3} ; r_{0}, \cdots, r_{4 j+6}$, it follows that $t^{4 j+6}, t^{4 j+7}, t^{4 j+8}, t^{4 j+9}$ are linearly expressible in terms of $r_{0}, \cdots, r_{4 j+6} ; r_{0}, \cdots, r_{4 j+7} ; r_{0}, \cdots, r_{4 j+7}$; 
$r_{0}, \cdots, r_{4 j+10}$. Thus $t^{k}$ is linearly expressible in terms of $r_{0}, r_{1}, \cdots, r_{k+1}$ for each $k \geqq 2$. The same is trivially true for $k=0$. The polynomial $r_{n}$ is orthogonal to every polynomial of degree $k$ not containing the first power of $t$ if $n>k+1$. The expression of $r_{n}$ in terms of the $q$ 's involves only $q_{n-1}, q_{n}, q_{n+1}, q_{n+2}, q_{n+3}$. By Schwarz's inequality, as on other occasions, the coefficients in the representation can not exceed 1 in magnitude. Since the $q$ 's are uniformly bounded [8] except near the ends of the interval $(a, b)$ and the point $t=0$, if the last belongs to the interval, this conclusion pertains to the r's also. The argument is for weight function unity with $t$ as variable of integration. It extends by Korous's theorem to unit weight function or any equivalent weight function with respect to arc length as independent variable, if the interval excludes the value $t=0$, that is to say, excludes the singular point of the curve; if this point is included, complications arise which will not be dealt with here.

5. The curve $x^{4}+y^{4}=1$. A non-rational curve of the fourth degree which possesses some interest by reason of its simplicity and symmetry is $x^{4}+y^{4}=1$. The question of approximation by means of polynomials on this curve, a sort of square-shouldered circle or round-cornered square, has been discussed elsewhere [3] with Bernstein's theorem as key to the problem of convergence. The treatment based on the boundedness of the polynomials of the orthonormal system is in some respects narrower in scope, but leads to results possessing greater precision in detail. As in the earlier sections, the present paper will be concerned merely with the property of boundedness, not with the processes of its application to proofs of convergence.

The independent monomials to be used for the construction of the orthonormal system are those not divisible by $y^{4}$, listed as follows; the significance of the brackets and parentheses will be explained presently:

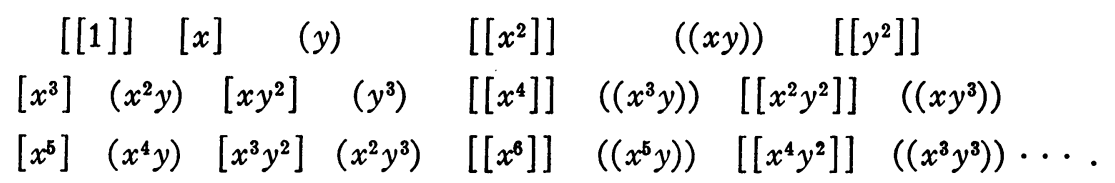

The monomials inclosed in double square brackets are even functions of $x$ and $y$ separately, and are positive in all four quadrants; those in single square brackets are even with respect to $y$ but odd with respect to $x$, and are positive in the first and fourth quadrants, negative in the second and third; those in single parentheses are positive in the first and second quadrants, negative in the third and fourth; and those in double parentheses are positive in the first and third quadrants and negative in the second and fourth. A monomial belonging to any one of the four sequences thus distinguished is orthogonal to any member of any of the other three sequences for integration with respect to arc length around a complete circuit of the curve. The complete orthonormal system for the curve can be constructed by applying Schmidt's process to each of the four sequences separately, and arranging the poly- 
nomials thus obtained in a single sequence according to the occurrence of their leading terms in (5) (cf. [10, pp. 346-347]).

If two polynomials are formed from terms belonging to a single one of the four sequences in (5), the same sequence in both cases, their product is an even function of both variables, and the integral of the product over the entire curve is four times the integral over the arc contained in the first quadrant. Orthonormal polynomials for the complete circuit are obtained by constructing orthonormal polynomials for the quadrant, and dividing each of these polynomials by 2 . It is sufficient to study the orthonormal systems belonging to the first quadrant.

In the first quadrant, the curve has the parametric representation

$$
x=(\cos \theta)^{1 / 2}, \quad y=(\sin \theta)^{1 / 2}, \quad 0 \leqq \theta \leqq \pi / 2 .
$$

In terms of $\theta$ the monomials with double brackets in (5) become

$$
1, \cos \theta, \sin \theta, \cos ^{2} \theta, \cos \theta \sin \theta, \cos ^{3} \theta, \cos ^{2} \theta \sin \theta, \cdots \text {. }
$$

Orthonormal combinations of these particular functions of $x$ and $y$ are orthogonal trigonometric sums in $\theta$ on the interval $(0, \pi / 2)$. If integration on the curve is to be performed with arc length as variable of integration and unit weight function, the weight function for integration with respect to $\theta$ is $d s / d \theta$, with

$$
\left(\frac{d s}{d \theta}\right)^{2}=\left(\frac{d x}{d \theta}\right)^{2}+\left(\frac{d y}{d \theta}\right)^{2}=\frac{\sin ^{2} \theta}{4 \cos \theta}+\frac{\cos ^{2} \theta}{4 \sin \theta}=\frac{\sin ^{3} \theta+\cos ^{3} \theta}{4 \sin \theta \cos \theta} .
$$

Since $\left(\sin ^{3} \theta+\cos ^{3} \theta\right)^{1 / 2}$ or its reciprocal has a positive lower bound and satisfies a Lipschitz condition for $0 \leqq \theta \leqq \pi / 2, d s / d \theta$ as weight function is equivalent from the point of view of Korous's theorem to $(\sin \theta \cos \theta)^{-1 / 2}$, and the problem is essentially that of the boundedness of the orthonormal trigonometric sums corresponding to the latter weight function $[6 ; \S 4]$. As a study of these particular trigonometric sums is not on record, however, the analysis must be continued further here.

Let $\phi=\theta-(\pi / 4)$, and let $u=\cos \phi, v=\sin \phi$. Orthonormal trigonometric sums in $\theta$ with weight $(\sin \theta \cos \theta)^{-1 / 2}$ are orthonormal trigonometric sums in $\phi$ with weight $2^{1 / 2}\left(\cos ^{2} \phi-\sin ^{2} \phi\right)^{-1 / 2}$. They are orthonormal polynomials in $u$ and $v$ on the arc of the circle $u^{2}+v^{2}=1$ for which $-\pi / 4 \leqq \phi \leqq \pi / 4$, that is, on the arc symmetric above and below the $u$-axis for which $2^{-1 / 2} \leqq u \leqq 1$, the weight function for integration with respect to $u$ being

$$
2^{1 / 2}\left(\cos ^{2} \phi-\sin ^{2} \phi\right)^{-1 / 2}|d \phi / d u|=\left[\left(1-u^{2}\right)\left(u^{2}-1 / 2\right)\right]^{-1 / 2} .
$$

For the purposes of Korous's theorem in the form applicable to ordinary polynomials, as distinguished from the trigonometric sums of the preceding paragraph (see [12, p. 157], [5, pp. 205-208]) this is equivalent to 


$$
[(1-u)(u-c)]^{-1 / 2}
$$

with $c=1 / 2^{1 / 2}$, since the factor $[(1+u)(u+c)]^{1 / 2}$ is positive and satisfies a Lipschitz condition over the range of values of $u$ in question.

Independent monomials in terms of $u$ and $v$ are

$$
1, u, v, u^{2}, u v, u^{3}, u^{2} v, \cdots,
$$

containing no power of $v$ higher than the first. As in similar situations which have been encountered elsewhere, the orthonormal system is obtained by forming separately and then combining into a single sequence the appropriate polynomials containing and not containing the factor $v$. The orthonormal polynomials independent of $v$, with the weight function (6), are essentially the cosine polynomials in terms of $u$ (see, for example, [5, pp. 149-150, 191]) adjusted by linear transformation of the independent variable to the interval $(c, 1)$, and are uniformly bounded throughout the closed interval; integration over the entire arc of the circle corresponds to be sure to integrating twice over the interval of values for $u$, but a constant factor in the weight function is immaterial as far as boundedness of the polynomials is concerned. The orthonormal polynomials containing $v$ as a factor are found by constructing the orthonormal polynomials in $u$ on the interval $(c, 1)$ for a weight function which is a constant multiple of

$$
v^{2}[(1-u)(u-c)]^{-1 / 2}=(1+u)(1-u)^{1 / 2}(u-c)^{-1 / 2}
$$

and multiplying each by $v$. With respect to Korous's theorem this function is equivalent to $(1-u)^{1 / 2}(u-c)^{-1 / 2}$ as weight, since $1+u$ has a positive lower bound and satisfies a Lipschitz condition on the range of integration.

The problem of boundedness associated with the last weight function is reduced by linear transformation of the independent variable to that for weight $u^{1 / 2}(1-u)^{-1 / 2}$ on the interval $0 \leqq u \leqq 1$. Let $c_{n}(z)$ be the normalized cosine polynomial of the $n$th degree for $-1 \leqq z \leqq 1$, let $u=z^{2}$, and let the odd polynomial $c_{2 n+1}(z)=z q_{n}(u)$. Then

$$
\begin{aligned}
\int_{-1}^{1}\left(1-z^{2}\right)^{-1 / 2} c_{2 m+1}(z) c_{2 n+1}(z) d z & =2 \int_{0}^{1}\left(1-z^{2}\right)^{-1 / 2} c_{2 m+1}(z) c_{2 n+1}(z) d z \\
& =\int_{0}^{1} u^{-1 / 2}(1-u)^{-1 / 2} \cdot z q_{m}(u) \cdot z q_{n}(u) d u \\
& =\int_{0}^{1} u^{1 / 2}(1-u)^{-1 / 2} q_{m}(u) q_{n}(u) d u ;
\end{aligned}
$$

the $q$ 's are the normalized orthogonal polynomials for weight $u^{1 / 2}(1-u)^{-1 / 2}$. The product $u^{1 / 2} q_{n}(u)=z q_{n}(u)=c_{2 n+1}(z)=\left(1 / \pi^{1 / 2}\right) \cos (2 n+1) \psi$ with $z=\cos \psi$ is uniformly bounded throughout the interval of values of $u$, and this means in the notation of the preceding paragraph that if the orthonormal poly- 
nomials for weight $(1-u)^{1 / 2}(u-c)^{-1 / 2}$ on the interval $(c, 1)$ are multiplied by $(1-u)^{1 / 2}$ the products are uniformly bounded throughout the interval. The same is true if the weight function is replaced by (7) [7, pp. 352-353]. The conclusion still holds if the products are further multiplied by the bounded factor $\pm(1+u)^{1 / 2}$, and the polynomials themselves by $\pm\left(1-u^{2}\right)^{1 / 2}=v$. In summary, the orthonormal polyniomials with weight $[(1-u)(u-c)]^{-1 / 2}$ for integration with respect to $u$ on the arc of the unit circle in the $(u, v)$-plane for which $u \geqq c$ are uniformly bounded on the entire arc, inclusive of its end points.

It will be noted that for the reasoning of the two preceding paragraphs the numerical value of $c$ is immaterial, beyond the restriction that $-1<c<1$.

In terms of an earlier notation, the reasoning just completed leads to the conclusion that the orthonormal trigonometric sums in $\theta$ on the interval $(0, \pi / 2)$ for weight $(\sin \theta \cos \theta)^{-1 / 2}$ and hence those for weight

$$
2^{-1}\left(\sin ^{3} \theta+\cos ^{3} \theta\right)^{1 / 2} /(\sin \theta \cos \theta)^{1 / 2}
$$

are uniformly bounded on the closed interval, and hence further that the orthonormal polynomials formed from the double-bracketed monomials in (5) for the first quadrant of the curve $x^{4}+y^{4}=1$, with arc length as variable of integration and unit weight function, are uniformly bounded on the entire arc.

The monomials with single brackets are respectively the products of those with double brackets by the factor $x$. The corresponding orthonormal polynomials are obtained by multiplying by $x$ the orthonormal polynomials in terms of the double-bracketed monomials for weight $x^{2}$. The polynomials last specified are the orthonormal trigonometric sums in $\theta$ on the interval $(0, \pi / 2)$ for the weight function which results from multiplication of (8) by $x^{2}=\cos \theta$. Since this factor is a trigonometric sum which is non-negative on the interval, the property of boundedness of the trigonometric sums is preserved where $\cos \theta \neq 0$ [1, pp. 806-807]; they are uniformly bounded throughout any closed interval of $(0, \pi / 2)$ from which the point $\theta=\pi / 2$ is excluded, and the polynomials in $x$ and $y$ are uniformly bounded on the arc except near the point $(0,1)$. Similarly, the orthonormal polynomials constructed from the monomials in single parentheses are uniformly bounded on the arc except in the neighborhood of the point $(1,0)$, and those based on the monomials in double parentheses are uniformly bounded if neighborhoods of both ends of the arc are avoided. With respect to mere boundedness, as distinguished from uniform boundedness, the quadrantal points are not exceptional, since in each case the polynomials with regard to which the question arises vanish there.

The orthonormal polynomials for the curve $x^{4}+y^{4}=1$, with arc length as variable of integration and unit weight function, are bounded at any single point of the curve, and are uniformly bounded except near the points where the curve crosses the coordinate axes. 
Even with respect to uniform boundedness the reservation concerning the points of intersection with the axes has no obvious appropriateness, since the curve is if anything smoother there than elsewhere. It is perhaps worth while, even at the expense of some additional labor, to verify that the reservation is in fact unnecessary: the orthonormal polynomials are uniformly bounded on the entire curve.

Let the coordinates be subjected to the linear transformation

$$
x=(\xi-\eta) / 2^{1 / 2}, \quad y=(\xi+\eta) / 2^{1 / 2},
$$

corresponding to a rotation of axes through $45^{\circ}$. Orthonormal polynomials in $x$ and $y$ are orthonormal polynomials of like degree in $\xi$ and $\eta$, with arc length as variable of integration and unit weight function in each case. The individual polynomials obtained by straightforward application of the Schmidt process will not be the same, but the polynomials of any specified degree in one pair of variables must be expressible on the curve in terms of those of the same degree in the other pair of variables by means of an orthogonal transformation [2, pp. 233-234], which does not affect the property of boundedness.

The equation of the curve in terms of the new variables is

$$
\xi^{4}+6 \xi^{2} \eta^{2}+\eta^{4}=2 \text {. }
$$

As independent monomials may be taken those not divisible by $\eta^{4}$. These can be arranged in four sequences, in accordance with the scheme illustrated in (5), so that any two monomials from different sequences are orthogonal to each other for integration over a complete circuit, and two polynomials formed from different sequences have the corresponding property of orthogonality. As far as polynomials from a single sequence are concerned, the integral of the product of two such polynomials from the same sequence around the entire curve is four times the integral over the first quadrant. It is sufficient again to study the boundedness of orthonormal systems formed for the first quadrant from the four sequences separately.

The expressions in $\xi$ and $\eta$ corresponding to the double-bracketed entries in (5) are

$$
1, \xi^{2}, \eta^{2}, \xi^{4}, \xi^{2} \eta^{2}, \xi^{6}, \xi^{4} \eta^{2}, \cdots
$$

With $u=\xi^{2}, v=\eta^{2}$, these are

$$
1, u, v, u^{2}, u v, u^{3}, u^{2} v, \cdots,
$$

a fundamental system of monomials for the hyperbola

$$
u^{2}+6 u v+v^{2}=2
$$

on which the point $(u, v)$ is situated if the coordinates $(\xi, \eta)$ satisfy $(9)$. As $(\xi, \eta)$ describes the first quadrant of the curve $(9),(u, v)$ traces the portion of 
the hyperbola in the first quadrant of the $(u, v)$-plane, an arc for which $0 \leqq u \leqq 2^{1 / 2}, 0 \leqq v \leqq 2^{1 / 2}$. The present $u$ and $v$ then are quite different from the variables previously designated by the same letters. If $s$ and $s^{\prime}$ denote arc length on the curves (9) and (10) respectively,

$$
\begin{gathered}
\left(\frac{d s^{\prime}}{d u}\right)^{2}=1+\left(\frac{d v}{d u}\right)^{2}=1+\left(\frac{u+3 v}{3 u+v}\right)^{2}, \\
d s^{2}=d \xi^{2}+d \eta^{2}=d u^{2} /(4 u)+d v^{2} /(4 v), \\
\left(\frac{d s}{d u}\right)^{2}=\frac{1}{4 u}+\frac{1}{4 v}\left(\frac{d v}{d u}\right)^{2}=\frac{1}{4 u}+\frac{1}{4 v}\left(\frac{u+3 v}{3 u+v}\right)^{2} .
\end{gathered}
$$

Unit weight function for integration with respect to $s$ means weight function $d s / d s^{\prime}$ for integration with respect to $s^{\prime}$, and

$$
\frac{d s}{d s^{\prime}}=\frac{\sigma}{(u v)^{1 / 2}}
$$

with

$$
\sigma=\frac{1}{2}\left[\frac{v(3 u+v)^{2}+u(u+3 v)^{2}}{(3 u+v)^{2}+(u+3 v)^{2}}\right]^{1 / 2} .
$$

For the range of variables considered, $u$ and $v$ and $s$ are single-valued functions of $s^{\prime} ; \sigma$ is bounded and has a positive lower bound, since $u$ and $v$ are nonnegative and do not vanish simultaneously on the arc in question; and

$$
\frac{d \sigma}{d s^{\prime}}=\frac{d \sigma}{d u} \frac{d u}{d s^{\prime}}=\left(\frac{\partial \sigma}{\partial u}+\frac{\partial \sigma}{\partial v} \frac{d v}{d u}\right) \frac{d u}{d s^{\prime}}
$$

is bounded, so that $\sigma$ satisfies a Lipschitz condition as a function of $s^{\prime}$. Hence the conditions for the application of Korous's theorem are fulfilled [6] to the effect that $d s / d s^{\prime}$ is equivalent to $1 /(u v)^{1 / 2}$ as weight function on the hyperbolic arc.

For the purposes of this paragraph and the next two, let

$$
x=u+v, \quad y=2^{-1 / 2}(-u+v) ; \quad u=\left(x-2^{1 / 2} y\right) / 2, \quad v=\left(x+2^{1 / 2} y\right) / 2 .
$$

The present $x$ and $y$ are not the same as the previous $x$ and $y$; any possibility of confusion is regarded as insignificant in comparison with the convenience of the use of the familiar letters. On the curve (10),

$$
\begin{aligned}
2=u^{2}+6 u v+v^{2}= & \left(x^{2}-2^{3 / 2} x y+2 y^{2}\right) / 4+6\left(x^{2}-2 y^{2}\right) / 4 \\
& +\left(x^{2}+2^{3 / 2} x y+2 y^{2}\right) / 4 \\
= & 2 x^{2}-2 y^{2}, \\
x^{2}-y^{2}= & 1 .
\end{aligned}
$$


The arc of $(10)$ in the first quadrant of the $(u, v)$-plane becomes the arc of $11)$, symmetric above and below the $x$-axis, for which $1 \leqq x \leqq 2^{1 / 2}$.

If $s^{\prime \prime}$ denotes length of arc on (11), $u$ and $v$ and $s^{\prime}$ and $s^{\prime \prime}$ are all singlevalued functions of each other on the arcs under consideration in the $(u, v)$ -

$\mathrm{d}(, y)$-planes. The change from $s^{\prime}$ to $s^{\prime \prime}$ as variable of integration involves introduction of a factor $d s^{\prime} / d s^{\prime \prime}$ in the weight function. It can be shown with some calculation, but with no difficulty in principle, that this factor satisfies the Korous conditions as a function of any one of the four variables named, $s^{\prime \prime}$ in particular, so that the weight function is still equivalent to

$$
1 /(u v)^{1 / 2}=2 /\left(x^{2}-2 y^{2}\right)^{1 / 2}
$$

for $s^{\prime \prime}$ as variable of integration. On the arc of (11), by an argument which has been used repeatedly in other situations, the orthonormal system for weight (12) is made up of polynomials in $x$ alone and polynomials containing the first power of $y$ as a factor, any member of one set being orthogonal to any member of the other set, inasmuch as the weight function is even with respect to $y$.

Integration of any even function of $y$ over the arc with respect to $s^{\prime \prime}$ amounts to integration twice with respect to $x$ over the interval $1 \leqq x \leqq 2^{1 / 2}$ after introduction of the factor

$$
\left|\frac{d s^{\prime \prime}}{d x}\right|=\left(1+\frac{x^{2}}{y^{2}}\right)^{1 / 2}=\frac{\left(2 x^{2}-1\right)^{1 / 2}}{\left(x^{2}-1\right)^{1 / 2}} .
$$

Since

$$
\begin{aligned}
\frac{1}{(u v)^{1 / 2}}\left|\frac{d s^{\prime \prime}}{d x}\right| & =\frac{2}{\left(2-x^{2}\right)^{1 / 2}} \frac{\left(2 x^{2}-1\right)^{1 / 2}}{\left(x^{2}-1\right)^{1 / 2}} \\
& =\frac{2\left(2 x^{2}-1\right)^{1 / 2}}{\left[\left(2^{1 / 2}+x\right)(x+1)\right]^{1 / 2}} \cdot \frac{1}{[(c-x)(x-1)]^{1 / 2}}
\end{aligned}
$$

with $c=2^{1 / 2}$, and since $2\left(2 x^{2}-1\right)^{1 / 2}\left[\left(2^{1 / 2}+x\right)(x+1)\right]^{-1 / 2}$ satisfies the Korous conditions on the interval $(1, c)$, the weight function for the orthonormal polynomials not containing $y$ is equivalent to $1 /[(c-x)(x-1)]^{1 / 2}$. The polynomials belonging to the last weight function are essentially the cosine polynomials adjusted to the interval $(1, c)$, and are uniformly bounded throughout the closed interval. The polynomials containing $y$ as a factor are to be obtained by forming the orthonormal system for weight

$$
\frac{y^{2}}{(u v)^{1 / 2}}\left|\frac{d s^{\prime \prime}}{d x}\right|=\frac{2\left(x^{2}-1\right)}{\left(2-x^{2}\right)^{1 / 2}} \frac{\left(2 x^{2}-1\right)^{1 / 2}}{\left(x^{2}-1\right)^{1 / 2}}=\frac{2(x+1)^{1 / 2}\left(2 x^{2}-1\right)^{1 / 2}}{\left(2^{1 / 2}+x\right)^{1 / 2}} \cdot \frac{(x-1)^{1 / 2}}{(c-x)^{1 / 2}}
$$

and multiplying each by $y$. The weight function is equivalent to 
$(x-1)^{1 / 2}(c-x)^{-1 / 2}$, and by adaptation of previous reasoning to the interval $(1, c)$ the products obtained on multiplication by $y$ are again uniformly bounded throughout the closed interval.

In the notation of the paragraphs preceding the last three, the orthonormal polynomials in $u$ and $v$ with weight $1 /(u v)^{1 / 2}$ for the arc of (10) belonging to the first quadrant of the coordinate system, and hence the orthonormal polynomials in even powers of $\xi$ and $\eta$ with unit weight function on the first quadrant of (9), are uniformly bounded over the ranges specified, the variable of integration in each case being arc length on the curve in question. The problem of boundedness for the other orthonormal sequences in $\xi$ and $\eta$, namely those involving respectively odd powers of $\xi$, odd powers of $\eta$, and odd powers of both variables, reduces to the corresponding question for the orthonormal sequences on $(10)$ with weights $(u / v)^{1 / 2},(v / u)^{1 / 2}$, and $(u v)^{1 / 2}$. Since these weights are products of $1 /(u v)^{1 / 2}$ by $u, v$, and $u v$, integral rational functions non-negative on the range of integration, the orthonormal polynomials in $u$ and $v$ are uniformly bounded except in the neighborhood of the points where the respective multipliers vanish. The proof, if not on record in detail, follows the pattern of those relating to polynomials in a single variable and trigonometric sums (see for example, [5, pp. 201-203], [1, pp. 806$807]$ ). The property of boundedness is naturally not impaired by the final inclusion of the factors $\xi, \eta$, or $\xi \eta$ in the polynomials of the various sequences.

The orthonormal polynomials on (9) with unit weight function are therefore uniformly bounded when the point $(\xi, \eta)$ is at a distance from the coordinate axes in the $(\xi, \eta)$-plane. That is to say, the orthonormal polynomials on the curve $x^{4}+y^{4}=1$ are uniformly bounded when $(x, y)$ is at a distance from the points of the curve where $x= \pm y$. But they are already known to be uniformly bounded in the neighborhood of these points, and everywhere on the curve except in the neighborhood of the points where $x y=0, \xi= \pm \eta$, which require no special consideration when the analysis is carried through in terms of the latter pair of variables. The polynomials are in fact uniformly bounded on the entire curve.

\section{BIBLIOGRAPHY}

1. D. Jackson, Orthogonal trigonometric sums, Ann. of Math. (2) vol. 34 (1933) pp. 799-814. 236.

2. - Orthogonal polynomials on a plane curve, Duke Math. J. vol. 3 (1937) pp. 228-

3. - Polynomial approximation on a curve of the fourth degree, Bull. Amer. Math. Soc. vol. 43 (1937) pp. 388-393.

4. - Orthogonal polynomials with auxiliary conditions, Trans. Amer. Math. Soc. vol. 48 (1940) pp. 72-81.

5. - Fourier series and orthogonal polynomials, Carus Monograph Series, no. 6, 1941.

6. - Generalization of a theorem of Korous on the bounds of orthonormal polynomials, Bull. Amer. Math. Soc. vol. 48 (1942) pp. 602-608.

7. - Boundedness of orthonormal polynomials on loci of the second degree, Duke Math. J. vol. 11 (1944) pp. 351-365. 
8. - Boundedness of orthonormal polynomials in one, two, and three variables, Trans. Amer. Math. Soc. vol. 58 (1945) pp. 167-183.

9. - The boundedness of orthonormal polynomials on certain curves of the third degree, Bull. Amer. Math. Soc. vol. 52 (1946) pp. 899-907.

10. F. Koehler, Systems of orthogonal polynomials on certain algebraic curves, Bull. Amer. Math. Soc. vol. 46 (1940) pp. 345-351.

11. Margaret Martin, Some new systems of orthogonal polynomials on algebraic curves, Thesis, University of Minnesota, not yet published.

12. G. Szegö, Orthogonal polynomials, Amer. Math. Soc. Colloquium Publications, vol. 23, 1939.

UNIVERSITY OF MINNESOTA,

Minneapolis, MinN. 